\title{
Osseointegration- Key Factors Affecting Its Success-An Overview
}

\author{
Dr. Naveen Reddy Vootla ${ }^{1}$,Dr. K. Varun Reddy ${ }^{2}$ \\ ${ }^{1}$ (Private Practitioner, India) \\ ${ }^{2}$ (Department of Prosthodontics, Narayana Dental College, India)
}

\begin{abstract}
During the past two decades, dental implants have been used extensively to achieve osseointergration for prosthetic rehabilitation of edentulism. For this, a surgical procedure is performed on patient to insert a foreign material i.e implant into the bone, after which a poorly organized woven bone is formed at the interface, thus having a relatively low inherent strength. After a period of 3 to 6 months, woven bone is replaced by lamellar bone which possess adequate strength for load bearing. This bone healing process is known as osseointegration. This process of osseointegration depends not only on implant related factors such as material, shape, topography and surface chemistry but also mechanical loading, surgical technique and patient variables such as bone quality and quantity. There are many materials and techniques present today to increase the rate of clinical success of implants but the ultimate long-term success of an implant is dependent upon the efforts of both the patient and dentist in maintaining the health of the peri-implant tissues. The purpose of this review is to enlight various factors that have significant affect on osseointegration
\end{abstract}

Keywords: Osseointegration, Factors Affecting Osseointegration, Implant Bone Interface.

\section{Introduction}

Endosseous implants are used in dentistry as restorative modality for missing teeth where they function as artificial roots onto which a dental prosthesis may be anchored. ${ }^{1}$ The discovery of osseointegration and its application to clinical dentistry by Professor Per- Ingvar Branemark and his colleagues is one of the most significant and important developments in dentistry. ${ }^{2}$ Following insertion of an implant, a poorly organized woven bone is formed at the interface, thus having a relatively low inherent strength. After a period of 3 to 6 months, woven bone is replaced by lamellar bone which possess adequate strength for load bearing. The end of this bone healing process is called osseointegration. Development of this interface is complex and involves numerous factors. These include not only implant related factors such as material, shape, topography and surface chemistry but also mechanical loading, surgical technique and patient variables such as bone quality and quantity. $^{3}$

\section{Key Factors For Successful Implant Osseointegration}

The successful outcome of any implant procedure is dependent on the interrelationship of the following: ${ }^{4}$

1. Biocompatibility of the Implant material

2. Macroscopic and microscopic nature of the implant surface

3. The status of the implant bed in both a health (non-infected) and a morphologic (bone quality) context

4. The surgical technique

5. The undisturbed healing phase

6. The subsequent prosthetic design and long term loading phase.

\section{Implant Materials}

Several organizations have provided guidelines for the standardization of implant materials. To date, a multinational survey by ISO indicated that titanium and its alloy are mainly used. The most widely used nonmetallic implants are oxidic, carbonic or graphitic oxide like materials. The major groups of implantable materials for dentistry are titanium and alloys, cobalt chromium alloys, austenitic Fe-Cr-Ni-Mo steels, tantalum, niobium and zirconium alloys, precious metals, ceramics and polymeric materials. ${ }^{5}$

\subsection{Titanium and titanium alloys}

Titanium is a metal that presents low weight high strength/weight ratio, low modulus of elasticity, excellent corrosion resistance, excellent biocompatibility and easy shaping and finishing. Because of these properties, it is the material most widely used in the manufacture of dental implants, in the commercially pure titanium (CpTi) form or as an alloy. The most frequently used alloy (titanium.6 aluminum-4 vanadium) is composed of $90 \%$ titanium, $6 \%$ aluminum (decreases the specific weight and improves the elastic modulus) and $4 \%$ vanadium (decreases thermal conductivity and increases the hardness). 
This reactive group of metals and alloys (with primary elements from reactive group metallic substances) form tenacious oxides in air or oxygenated solutions. Titanium oxidizes (passivates) on contact with room temperature air and normal tissue fluids. This reactivity is favourable for dental implant devices in the absence of interfacial motion or adverse environmental conditions as this passivated (oxidized) surface condition minimizes bio-corrosion phenomena. Some reports show that the oxide layer tends to increase in thickness under corrosion testing and that breakdown of this layer is unlikely in aerated solution. ${ }^{5}$

\subsection{Cobalt-Chromium-Molybdenum based alloys}

In general, the as-cast cobalt alloys are the least ductile of the alloy systems used for dental surgical implants and bending of finished implants should be avoided. Because many of these alloy devices have been fabricated by dental laboratories, all aspects of quality control and analysis for surgical implants must be followed during alloy selection, casting, and finishing. When properly fabricated, implants from this alloy group have shown to exhibit excellent biocompatibility profiles. ${ }^{5}$

\subsection{Iron-Chromium-Nickel Based alloys}

This alloy is used most often in a wrought and heat-treated metallurgic condition, which results in a high-strength and high-ductility alloy. The ramus blade, ramus frame, stabilizer pins (old), and some mucosal insert systems have been made from the iron-based alloy. Of the implant alloys, this alloy is most subject to crevice and pitting biocorrosion, and care must be taken to use and retain the passivated (oxide) surface condition. Because this alloy contains nickel as a major element, use in patients allergic or hypersensitive to nickel should be avoided, in addition, if a stainless steel implant is modified before surgery, then recommended procedures call for repassivation to obtain an oxidized (passivated) surface condition to minimize in vivo biodegradation. 5

\subsection{Ceramics}

Ceramics are inorganic, non-metallic and non-polymeric materials manufactured by compacting and sintering at elevated temperatures. They can be divided into metallic oxides or other compounds. Oxide ceramics were introduced for surgical implant devices because of their inertness to biodegradation, high strength, physical characteristics such as color and minimal thermal and electrical conduction and a wide range of material specific elastic properties. In many cases, however, the low ductility or inherent brittleness has resulted in limitations. 5

\subsection{Aluminum, Titanium and Zirconium oxides}

High strength ceramics from aluminum, titanium, and zirconium oxides have been used for root form, endosteal plate form, and pin type of dental implants. The compressive, tensile and bending strengths exceed the strength of compact bone by three to five times. These properties, combined with high modulus of elasticity and especially with fatigue and fracture strengths, have resulted in specialized design requirements for these classes of biomaterials and the relative cost for manufacturing. The aluminium, titanium and zirconium oxide ceramics have a clear, white, cream, or light-gray color, which is beneficial for applications such as anterior root form devices. Schulte and Heinke developed a technique whereby aluminum oxide implants are inserted into the jaw immediately after the tooth has been extracted. These implants are commercially available in a modified form as Frialit Ceramic implants and have been used mainly for single tooth replacements. ${ }^{6}$

\subsection{Zirconia}

Zirconia $(\mathrm{ZrO} 2)$ is a ceramic material used in implantology because of its biocompatibility, esthetics (because its color is similar to the teeth), and mechanical properties which are better than alumina. Implants produced with $\mathrm{ZrO} 2$ are biocompatible, bioinert and radiopaque and they present a high resistance to corrosion, flexion and fracture. This material is reported to present a contact with bone and soft tissue similar to that observed in titanium implants and it can be used to produce an entire implant or as a coating. The interface is composed by a proteoglycan layer, which is thicker than titanium (ranging from 300 to $500 \mathrm{~A}^{\circ}$ and 200 to 400 $\mathrm{A}^{\mathrm{o}}$ respectively). ${ }^{5}$

\subsection{Carbon compounds}

Carbon compounds are often classified as ceramics because of their chemical inertness and absence of ductility. However, they are conductors of heat and electricity. Vitreous carbon implants have a core of stainless steel that is covered by $99.99 \%$ pure carbon. A combination of design, material, and application limitations resulted in a significant number of clinical failures and the subsequent withdrawal of this device from clinical use. Ceramic and carbonitic substances continue to be used as coatings on metallic and ceramic materials. 
Advantages of coatings include tissue attachment; components that are normal to physiological environments; regions that serve as barriers to elemental transfer heat, or electrical current flow control of color; and opportunities for the attachment of active biomolecules or synthetic compounds. ${ }^{5}$

\section{Implant Surface}

\subsection{Macrogeometry}

The macrodesign or shape of an implant has an important bearing on the bone response; growing bone concentrates preferentially on protruding elements of the implant surface such as ridges, crests, teeth, ribs or the edge of the threads that apparently act as stress risers when load is transferred. The shape of the implant determines the surface area available for stress transfer and governs the initial stability of the implant. Smooth sided cylindrical implants provide ease in surgical placement. However, the bone to implant interface is subjected to significantly larger shear conditions. In contrast, a smooth sided tapered implant allows for a component of compressive load to be delivered to the bone to implant interface, depending on the degree of taper. The larger the taper, the greater the component of compressive load delivered to the interface. The amount of taper cannot be greater than $30^{\circ}$ or the implant body length is reduced significantly along with the immediate fixation required for initial healing. In addition, the greater the taper, of the smooth sided implant, the less the overall surface area of the implant body. Threaded (or plateaued) implants with circular cross-section

(Figure 1) provide for ease of surgical placement and allow for greater functional surface area (optimization to transmit compressive load to bone-to-implant interface). In addition, a threaded implant is easily rigidly fixated initially to limit micromovement during wound healing. A smooth sided cylinder depends on a coating or microstructure for load transfer to bone. This surface treatment may also be applied to a screw or plateau design increasing the functional surface from design and surface treatment conditions. 7

Unlike a cylinder implant, a tapered threaded implant serves no functional surface area advantage. The thread shape bears the compressive and tensile loads. The tapered thread has less surface area than a parallel threaded implant body. The tapered threaded implant cannot be unthreaded once seated to place the crest module in a more ideal prosthetic position. A tapered threaded implant most often has less deep threads because the outer diameter continues to decrease. Although tooth roots taper as they proceed to the apex, the threaded implant has little advantage and many disadvantages to follow the tooth root design. ${ }^{7}$

\subsubsection{Implant width}

Over the past 5 decades of endosteal implant history, implants gradually have increased in width. The pin implants provides for more compressive load transfer, which is particularly important in D3 and D4 bone.

The V-shape and reverse buttress had similar stress values. (Figure 2) The square thread had less stress in compressive and more importantly shear forces. The square thread exhibited higher reverse torque values after initial healing whereas reverse buttress and V-shape were similar. Thread shape may alter the functional load conditions and influence the type of force transmitted to bone. The greater the thread depth, the greater the surface area of the implant, if all other factors are equal. The reverse buttress thread of SteriOss has a $0.24 \mathrm{~mm}$ thread depth. The thread depth of most $\mathrm{V}=$ shaped threads is $0.375 \mathrm{~mm}$. The square thread of BioHorizons has a $0.42 \mathrm{~mm}$ thread depth. ${ }^{7}$

\subsubsection{Implant length}

As the length of an implant increases, so does the overall total surface area. The strength of the bone and the intimate contact between the bone and implant provide resistance to lateral loading. Attempting to engage the opposing cortical plate and preparing a longer osteotomy may result in overheating of the bone. Once the implant-bone interface is formed, excessively long implants do not receive stress transfer in the apical region and therefore are not needed.

In general, the use of short implants has not been recommended because the belief is that occlusal forces must be dissipated over a large implant area to preserve the bone. Less favourable success rates for shorter implants were observed in clinical studies. Overall, the shorter and smaller diameter implants had lower survival rates than their longer or wider counterparts. Longer implants have been suggested to provide greater stability under lateral loading conditions. However, increasing the length beyond a certain dimension may not reduce force transfer proportionately. ${ }^{7}$

\subsection{Microdesign}

The quest was for biocompatible if not bioactive surfaces. Surface modification is achieved through additive or subtractive processes. Titanium, preferably commercially pure titanium became the standard for endosseous implants both in orthopaedics and in Implantology. Titanium is a very reactive material that would 
not become integrated in tissues however its instantaneous surface oxidation creates a passivation layer of titanium oxides which have ceramic like properties making it very compatible with tissues.

\subsubsection{Sand blasting}

Sandblasting the metal core with gritting agents creates these modified surfaces. This process is influenced by the number and the speed of the rotations to which the implant is submitted as well as by the pressure and the size of the particles used. The blasting procedure is performed with the aim of increasing the irregularity of the surface of the implant, using agents such as aluminum oxide $\left(\mathrm{Al}_{2} \mathrm{O}_{3}\right.$, also called alumina) and titanium dioxide $\left(\mathrm{TiO}_{2}\right)$. The analyses of different implant surfaces revealed that sandblasted samples showed the largest variability in surface appearance. Sandblasting has been shown in some studies to allow the adhesion, proliferation, and differentiation of osteoblasts. On the other hand, fibroblasts were found to adhere with more difficulty to this surface; this could limit the soft tissue proliferation and potentially benefit bone formation. Alternatives to blasting with $\mathrm{Al}_{2} \mathrm{O}_{3}$ particles have also been tested. Blasting a surface with $\mathrm{TiO}_{2}$ particles was proposed to promote a modification on the implant by using a component of the oxide layer naturally formed around titanium implants. ${ }^{5}$

\section{Plasma sprayed surfaces}

Plasma-sprayed implants are prepared by spraying molten metal on the titanium base which results in a surface with irregularly sized and shaped valleys, pores and crevices, increasing the microscopic surface area by 6 to 10 times. This topography may improve the fixation of implants by the growth of bone into the coating, forming a mechanical interlock. (Figure 3)

\subsubsection{Titanium Plasma Spray}

The titanium plasma spray (TPS) surface has been reported to increase the surface area of the boneimplant interface and acts similarly to a three-dimensional surface, which may stimulate adhesion osteogenesis. The surface area increase has been reported to be as great as $600 \%$. Although tremendous increase in total surface area occurs at the microscopic level, the actual load-bearing capability of the coating increases the functional area by $25 \%$ to $30 \%$. Porous surfaces in the range of TPS (150 to 400 micro meters) also increase the tensile strength of the bone- implant interface, resist shear forces and improve load transfer. The increased surface roughness may also improve the initial fixation of the implant, especially in softer bone. (Figure 4) Some evidence indicates that the interface may form faster but no consensus exists regarding whether that may shorten clinical healing times. ${ }^{5}$ One disadvantage of using the plasma-sprayed implants is the detachment of titanium after implant insertion.

\subsubsection{Acid etched surfaces}

Acid-etching a titanium base was proposed to modify the implant surface without leaving the residues found after the sandblasting procedure, to avoid the non-uniform treatment of the surface, and to control the loss of metallic substance from the body of the implant. This is performed using baths of hydrochloric acid $(\mathrm{HCl})$, sulfuric acid $\left(\mathrm{H}_{2} \mathrm{SO}_{4}\right)$, $\mathrm{HF}$ and nitric acid $\left(\mathrm{HNO}_{3}\right)$ in different combinations. The roughness before etching, the acid mixture, the bath temperature and the etching time all affect the acid etching process. ${ }^{5}$

A dual acid-etched technique has been proposed to produce a microtextured (instead of a macrotextured) surface, which could be more predisposed to achieve desirable results. This is because higher adhesion of platelet genes and higher expression of extracellular genes were observed in this dual acid-etched surface. ${ }^{5}$

Osseotite Implant: is treated in a dual acid etching procedure using hydrochloric and sulphuric acids. However, top part of the implant is left as machined. The Osseotite implant has been claimed to show - de novo bone formationll. Several investigations with a 3-6 year follow up reported success/survival rates between $95 \%-99 \%{ }^{8}$

\subsubsection{Sand blasted and acid-etched}

In 1990s the study of a modified surface resultant from blasting (to produce a macrotexture) followed by acid etching (to produce a final microtexture) showed promising results. The resultant surface was constituted by uniformly scattered gaps and holes and it appeared to be slightly less rough than the plasmasprayed surface which presented a deeply irregular texture that provided a less favorable environment for cell spreading. ${ }^{5}$

Sandblasted and acid-etched implants tend to promote greater osseous contact at earlier time points compared with plasma-sprayed coated implants. This conclusion was derived from a dog study in which the test surface was prepared by blasting with 250 to 500 micrometers carborundum particles, and the acid etching was done with $\mathrm{HCl}$ and $\mathrm{H}_{2} \mathrm{SO}_{4}$. Sandblasting can be performed using different abrasive particles. For example, the surface obtained from acid etching and sandblasting with $\mathrm{ZrO}_{2}$ particles is reported to favor a better bone deposition as compared with plasma-sprayed and turned surfaces. ${ }^{5}$ SLA Implant surface was clinically 
introduced in 1997. Alkaline phosphatase activity in osteoblast-like cells is greater on SLA surfaces than on TPS surfaces. ${ }^{8}$

\subsubsection{Anodized surface}

The oxidation process has been used in dental implants to change the characteristics of the oxide layer and consequently to improve the biocompatibility of the surface. The advantage is to modify the surface without depositing grit particles. Anodized surfaces are prepared by applying a voltage on the titanium specimen immersed in an electrolyte. The resultant surface presents micropores of variable diameters and demonstrates lack of cytotoxicity; moreover, cell attachment and proliferation are enhanced as compared with turned surfaces. ${ }^{5}$ (Figure 5)

Ti Unite Implants: the surface is anodized, i.e. it has been manufactured by electrochemical anodic oxidation in galvanostatic mode using electrolytes. The surface has a relatively thin oxide layer (a few hundred nanometres) and is minimally rough (0.5-1.0 micrometres) in the upper region, whereas the apical region displays an oxide thickness in the range of more than and a roughness of more than 2 micrometres. ${ }^{8}$

\subsubsection{Lasers}

An advantage of lasers in surface modification is that laser has the property of melting surface layer locally. In addition, laser processing is contactless and the thermal, mechanical deformation of the substrate is generally low. Following types of lasers are used: CO2 lasers and Nd-YAG laser. To embed a new phase in a substrate by means of laser processing the new material can be repositioned on the substrate. However, to melt the substrate the heat has to be transported through the pre-positioned powder slurry. If the melting point of both the materials does not differ to a large extent a reasonable degree of mixture may occur. If this is undesirable, the possibility of powder injection should be considered. ${ }^{9}$

\subsubsection{Tricalcium Phosphate Coatings}

By coating a metallic implant with Tricalcium phosphate (TCP), an implant is produced that is biocompatible, bioreactive and partially biodegradable. While TCP does not induce new bone formation, it does have Osteoconductive properties that act as a scaffold or nidus for new bone in growth. It also forms a chemical bio reactive bone with the calcium and phosphorus in bone. On implantations the TCP ceramic is partially resorbed by solution-mediated dissolution and macrophage phagocytosis. The ingrowth of bone into the resorption voids and pores results in a primary, mechanical anchoring of the implant. Therefore, by coating the implant with TCP, osseointegration is enhanced by providing a bioreactive chemical bond with bone in addition to a physical interlocking within the resorptive cavities. ${ }^{4}$

\subsubsection{Hydroxyapatite coatings}

Hydroxyapatite coatings have a similar roughness and increase in functional surface area as TPS. A direct bone bond shown with HA coating and the strength of the HA-to-bone interface is greater than titanium to bone and even greater than TPS to bone. In addition, accelerated interfacial bone formation and maturation have been observed in dogs. An initial implant-to-bone interface contact is essential for a predicable interface to form. The space or gap between the implant and bone may affect the percentage of bone contact after healing. Gap healing may be enhanced by the HA coating. The corrosion rate of metal is also reduced, which is more significant for cobalt chrome alloys. ${ }^{5}$

Macroporous hydroxyapatite forms have failed because of failure of tissue to fill the porous implant material completely which can lead to infection with dehiscence and loss of implant. The carved surface is generally very rough because of the macroporous nature of the blocks thus making them more likely to traumatize the overlying mucoperiosteum and prone to subsequent breakdown and wound dehiscence. Moreover, dense hydroxyapatite is difficult to carve to the desired shape. The new microporous hydroxyapatite may overcome these problems and appears to be biocompatible within bone. ${ }^{10}$ Porous Corralline HA showed ingrowth of bone into the interconnected porosity. ${ }^{11} \mathrm{HA}$ surfaces seem to be conducive to the morphogenic activities of osteogenic cells. One result of these activities is the deposition of bone tissues directly on to the surface of implanted HA, partly as a result of nucleation and epitaxial growth. ${ }^{12}$

\subsection{Capcitively Coupled Electric Field (CCEF)}

\section{Other Factors Promoting Osseointegration}

CCEF treatment effectively stimulated osteogenesis near the implant by generating undifferentiated mesenchymal cells. It has been believed that functional loading on an implant restoration in the early period after implant placement prevents osseointegration in the nearby bone. However, by the applicaiton of CCEF after implant placement, shortens the recovery period of normal occlusal function. ${ }^{13}$ 


\subsection{Bovine Osteogenic Protein}

Osteogenic protein inserted into unmodified sockets with implants may significantly shorten the time interval between tooth extraction and osseointegration of the implant and thereby reduce the necessary period of total or partial edentulism. In addition, this treatment may expand the use of implant therapy and enhance success rates by eliminating a surgical procedure, reducing the amount of bone lost after tooth extraction, permitting the insertion of longer implants and minimizing prosthetic compromises associated with alveolar ridge resorption. ${ }^{14}$

\section{Figures}

Figure 1: Threaded implant with cylindrical configuration.

Figure 2: A) V-Thread, B) buttress thread, C) reverse buttress thread, D) square thread.

Figure 3: Schematic diagram of plasma spraying.

Figure 4: Titanium plasma spray surface. Close adaptation of bone to implant surface. No gaps or connective tissue present at the bone-implant interface.

Figure 5: Anodised surface. Many newly formed bony trabeculae are present at the interface.

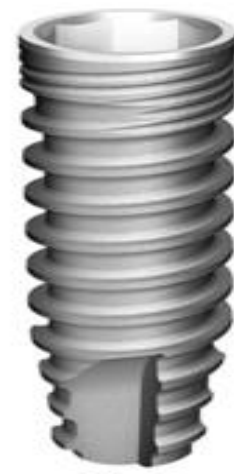

Fig 1
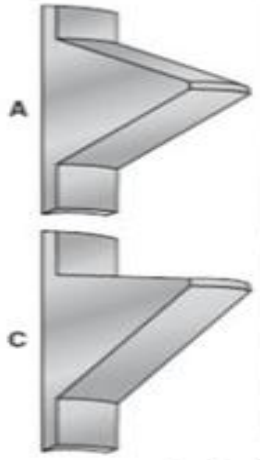

Fig 2
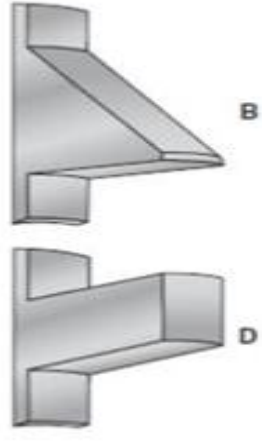

2

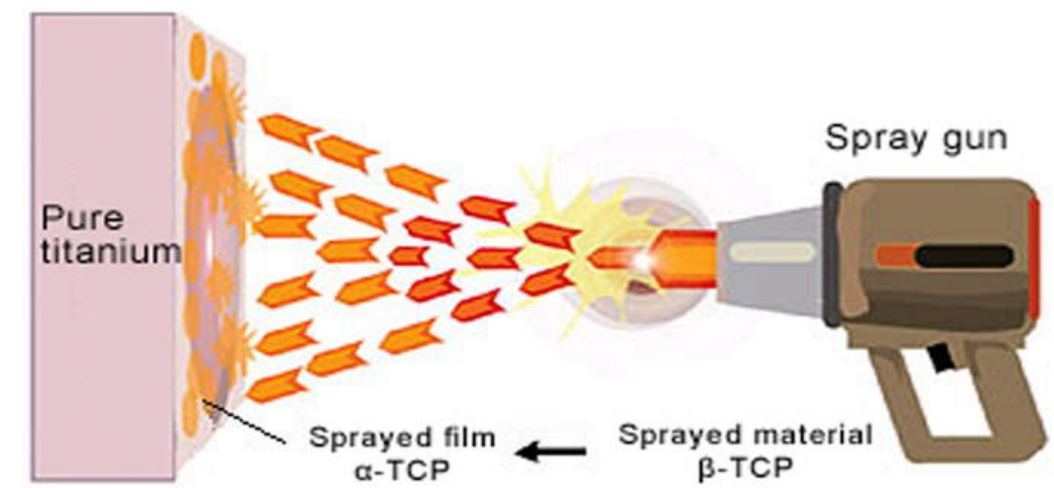

Fig 3

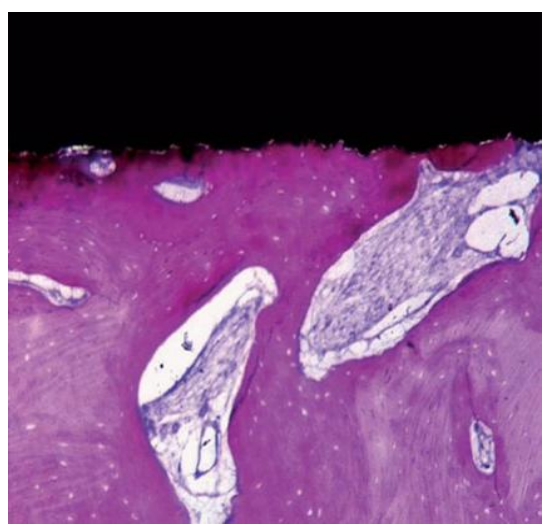

Fig 4

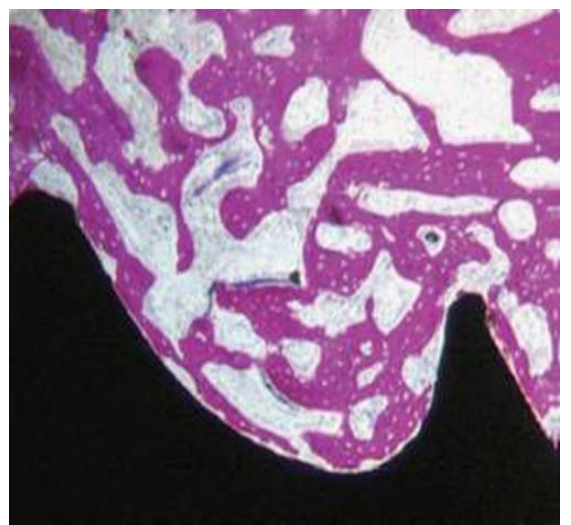

Fig 5 


\section{Summary}

The term-Osseointegration was coined by Dr Per-Ingvar Branemark, Professor at the Institute for Applied Biotechnology, University of Goteborg, Sweden in the year 1985. It is defined as a direct bone deposition on implant surfaces at the light microscopic level. This functional unit able to transmit occlusal forces to the alveolar bone has also been described as functional ankylosis (Schroeder). Osseointegration, once looked upon with scepticism, is now considered as a frequently occurring, primitive foreign body reaction to an implanted material. Osseointegration mainly depends on the quality and quantity of the available bone. Various factors influence the process of osseointegration which include biocompatibility of the implant material, surface topography of the implant, the surgical protocol followed and on the loading of the implants. Systemic and local factors also influence osseointegration. Clinical results can be improved by using the newer materials, designs, surgical techniques and loading protocols by using evidence based approach.

\section{References}

[1]. Sumiya Hobo. Osseointegration and occlusal rehabilitation; History of endosteal implants; Quintessence publishing; 22.

[2]. Branemark, Hansson B et al. Intraosseous anchorage of dental prosthesis1.experimental studies. Scand J Plast Reconstr Surg. 1970; 4 (1): 19-34

[3]. Hutton J, Heath M, Chai J, Damett J. Factors relating to success and failure rates at 3 year follow up in a multicentre study of overdentures supported by Branemark implants. Int J Oral Maxillofac Implants. 1995; 10 (1): 33-42.

[4]. Albrektsson T, Zarb G and Worthington P. The long term efficacy of currently used dental implants: A review and proposed criteria of success. Int J Oral Maxillofac Implants, 1986; 1: 11-25.

[5]. Carl E. Misch. Contemporory implant dentistry; Rational of dental implants; $3^{\text {rd }}$ edition, Elsevier publication; 3-4.

[6]. Zetterqvist $\mathbf{L}$, Anneroth $\mathbf{G}$ and Nordenram A. Tissue Integration of $\mathrm{Al}_{2} \mathrm{O}_{3}$-Ceramic Dental Implants. Int Oral Maxillofac Implants. 1991; 6: 285-293.

[7]. Misch CE. Dental implant prosthodontics. s.1. : Mosby, 2nd edition, 1999

[8]. Albrektsson T and Wennerberg A. Oral Implant Surfaces: Part 2-Review focusing on clinical knowledge of different surfaces. Int J Prosthodont. 2004; 17: 544-564.

[9]. Chaturvedi T P. Implantology made easy. s.l. : Jaypee, 1st edition, 2008.

[10]. Smith KG, Franklin CD, Noort R V et al. Tissue response to the implantation of 2 new machinable calcium phosphate ceramics. Int J Oral Maxillofac Implants. 1992; 7: 395-400.

[11]. Hansen EH, Worsaae $\mathbf{N}$ and Lemons J E. Histologic response after implantation of porous hydroxyapatite ceramic in humans. Int Oral Maxillofac Implants. 1990; 5: 255-263.

[12]. Bagambisa FB. The Interaction of Osteogenic cells with hydroxyapatite implant materials-In Vitro and In Vivo. Int J Oral Maxillofac Implants. 1990; 5: 217-226.

[13]. Shigino T, Ochi M, Hirose Y et al. Enhancing osseointegration by capacitively coupled electric field: a pilot study on early occlusal loading in the dog mandible. Int J Oral Maxillofac Implants. 2001; 16: 841-850.

[14]. Rutherford RB, Sampath K, Rueger DC, Taylor TD. Use of Bovine Osteogenic protein to promote rapid Osseointegration of Endosseous Dental Implants. Int J Oral Maxillofac Implants. 1992; 7: 297-301. 